\title{
Bacteriology, antibiotic resistance and risk stratification of patients with culture-positive, community-acquired pleural infection
}

\author{
Marianthi Iliopoulou ${ }^{1}$, Vasileios Skouras ${ }^{2}$, Zoe Psaroudaki ${ }^{3}$, Magda Makarona ${ }^{4}$, Evangelos Vogiatzakis ${ }^{4}$, \\ Eleni Tsorlini ${ }^{5}$, Eleni Katsifa ${ }^{5}$, Dionisios Spyratos ${ }^{6}$, Dimitra Siopi ${ }^{6}$, Ourania Kotsiou ${ }^{7}$, Stelios Xitsas ${ }^{8}$, \\ Maria Martsoukou', Ioanna Sigala ${ }^{10}$, Ioannis Kalomenidis ${ }^{10}$ \\ ${ }^{1} 7$ th Respiratory Medicine, Athens Chest Hospital "Sotiria”, Athens, Greece; ${ }^{2}$ Department of Pulmonary Medicine, 401 General Army Hospital, \\ Athens, Greece; ${ }^{3}$ Department of Clinical Microbiology, "Evangelismos" Hospital, Athens, Greece; ${ }^{4}$ Microbiology Laboratory and National Reference \\ Center for TB, Athens Chest Hospital "Sotiria”, Athens, Greece; ${ }^{5}$ Department of Microbiology, “G. Papanikolaou” Hospital, Aristotle University \\ of Thessaloniki, Thessaloniki, Greece; 'Department of Pulmonary Medicine, "G. Papanikolaou” Hospital, Aristotle University of Thessaloniki, \\ Thessaloniki, Greece; ${ }^{7}$ Department of Respiratory Medicine, University of Thessaly, Larissa, Greece; ${ }^{8}$ Department of Microbiology, University \\ Hospital of Larissa, Larissa, Greece; ${ }^{9}$ Department of Microbiology, "Sismanoglion” General Hospital, Athens, Greece; ${ }^{10} 1$ st Department of Critical \\ Care and Pulmonary Medicine, National and Kapodistrian University of Athens, "Evangelismos" Hospital, Athens, Greece \\ Contributions: (I) Conception and design: I Kalomenidis, M Iliopoulou; (II) Administrative support: I Kalomenidis; (III) Provision of study materials \\ or patients: M Iliopoulou, V Skouras, Z Psaroudaki, M Makarona, E Vogiatzakis, E Tsorlini, E Katsifa, D Spyratos, D Siopi, O Kotsiou, S Xitsas, \\ M Martsoukou, I Sigala; (IV) Collection and assembly of data: M Iliopoulou, V Skouras, Z Psaroudaki, M Makarona, E Vogiatzakis, E Tsorlini, E \\ Katsifa, D Spyratos, D Siopi, O Kotsiou, S Xitsas, M Martsoukou, I Sigala; (V) Data analysis and interpretation: M Iliopoulou, I Kalomenidis; (VI) \\ Manuscript writing: All authors; (VII) Final approval of manuscript: All authors. \\ Correspondence to: Marianthi Iliopoulou. 7th Respiratory Medicine Department, Athens Chest Hospital "Sotiria”, 152 Mesogeion Avenue, 11527
} Athens, Greece. Email: dr.mar.iliopoulou@gmail.com.

Background: Community-acquired pleural infection (CAPI) is a growing health problem worldwide. Although most CAPI patients recover with antibiotics and pleural drainage, $20 \%$ require surgical intervention. The use of inappropriate antibiotics is a common cause of treatment failure. Awareness of the common causative bacteria along with their patterns of antibiotic resistance is critical in the selection of antibiotics in CAPI-patients. This study aimed to define CAPI bacteriology from the positive pleural fluid cultures, determine effective antibiotic regimens and investigate for associations between clinical features and risk for death or antibiotic-resistance, in order to advocate with more invasive techniques in the optimal timing.

Methods: We examined 158 patients with culture positive, CAPI collected both retrospectively (2012-2013) and prospectively (2014-2018). Culture-positive, CAPI patients hospitalized in six tertiary hospitals in Greece were prospectively recruited $(\mathrm{N}=113)$. Bacteriological data from retrospectively detected patients were also used $(\mathrm{N}=45)$. Logistic regression analysis was performed to identify clinical features related to mortality, presence of certain bacteria and antibiotic resistance.

Results: Streptococci, especially the non-pneumococcal ones, were the most common bacteria among the isolates, which were mostly sensitive to commonly used antibiotic combinations. RAPID score (i.e., clinical score for the stratification of mortality risk in patients with pleural infection; parameters: renal, age, purulence, infection source, and dietary factors), diabetes and CRP were independent predictors of mortality while several patient co-morbidities (e.g., diabetes, malignancy, chronic renal failure, etc.) were related to the presence of certain bacteria or antibiotic resistance.

Conclusions: The dominance of streptococci among pleural fluid isolates from culture-positive, CAPI patients was demonstrated. Common antibiotic regimens were found highly effective in CAPI treatment. The predictive strength of RAPID score for CAPI mortality was confirmed while additional risk factors for mortality and antibiotic resistance were detected. 
Keywords: Clinical epidemiology; empyema; pleural disease; respiratory infections; bacteria

Submitted Sep 02, 2020. Accepted for publication Nov 06, 2020.

doi: $10.21037 /$ jtd-20-2786

View this article at: http://dx.doi.org/10.21037/jtd-20-2786

\section{Introduction}

Pleural infection represents the bacterial invasion of the pleura, most commonly after translocation of bacteria from the infected lung to the pleural space of patients with pneumonia (1). When the pleural infection is due to community bacteria, it is called CAPI and differs significantly in bacteriology and mortality from the hospital-acquired one (2). Approximately $50 \%$ of the patients with community-acquired pneumonia develop parapneumonic effusion and $10 \%$ of these effusions become infected $(1,3-5)$. The management of pleural infection includes the administration of proper antibiotics combined with drainage of the infected pleural fluid (6).

Although the majority of patients recovers with this treatment, $20 \%$ of them eventually require surgical intervention to treat their infection and 20\% die within 12 months from diagnosis (1). Current guidelines support the surgical intervention as a rescue therapy in cases of unresponsive antimicrobial treatment or need for decortication due to advanced fibrosis $(6,7)$. Some countries advocate the approach of early surgical intervention. Although, it is crucial for the patient's survival to find the appropriate time for surgery based on patient's clinical, imaging and laboratory characteristics and always in relation to age and co-morbidities (8-10).

The use of inappropriate antibiotics along with misdiagnosis, drainage delay and chest tube malposition represent the most common causes of failure of the initial treatment $(6,11)$. However, the selection of proper antibiotics is rarely an easy task; the fact that the bacteriology of pleural infection differs significantly from that of pneumonia together with the low (40-60\%) sensitivity of pleural fluid cultures and the absence of culture results at the time of diagnosis, require the institution of antibiotic treatment in the absence of microbiological guidance in most cases. Thus, it is of major importance to unveil the bacteriology of pleural infection, which not only depends on the origin of the infection (community-versus hospital-acquired) but also presents significant geographic variation $(2,6,12-14)$.

\section{Study endpoints}

The main purpose of this study was to determine the bacteriology and record the sensitivity test results of the bacterial isolates from CAPI-patients. We also aimed to examine whether certain clinical characteristic could predict the causative bacteria, resistance to certain antibiotics or mortality risk in order to specify the timing for more invasive solutions.

We present the following article in accordance with the STROBE reporting checklist (available at http://dx.doi. org/10.21037/jtd-20-2786).

\section{Methods}

\section{Study design}

This was a multicenter observational study that evaluated consecutive adult CAPI-patients who were hospitalized in six tertiary hospitals in Greece from January 2014 to May 2018 and had a positive pleural fluid culture (prospective arm of the study). Patients from this study arm, alive at three months post-discharge were identified by phone-call interview (Figure S1).

However, to expand the input for the determination of CAPI bacteriology, the laboratory database in four of the six involved hospitals was additionally, retrospectively reviewed to detect consecutive patients fulfilling the inclusion criteria (from January 2012 to December 2013, retrospective arm of the study). Only data regarding the isolated bacteria and their susceptibility test results were used from these patients.

\section{Inclusion/exclusion criteria}

Eligible patients (18 years old and above) were prospectively recruited by the local investigators, who were alarmed by the microbiology laboratory each time a positive pleural fluid culture was detected. As a common practice in the departments participating in the present study, pleural fluid samples are routinely submitted for culture, in every patient 
with pleural effusion of unknown etiology undergoing diagnostic thoracentesis regardless the clinical suspicion of pleural infection or the appearance of the fluid. Patients with tuberculous pleuritis, hospital-acquired (i.e., onset after more than 48 hours upon hospitalization) or healthcare-associated pleural infection (i.e., those manifested at hospital admission or within 48 hours after admission in patients complying with one of the following criteria: residence in a long-term-care facility, hospitalization within the last four weeks and receipt of intravenous medical therapy within the previous 30 days) and empyema caused by extra-thoracic sepsis or trauma, were excluded from the study. Inclusion and exclusion criteria for the retrospective arm of the study were the same as for the prospective one.

\section{Factors of analysis}

Patient demographics, co-morbidities, clinical and imaging findings at diagnosis, RAPID score (i.e., clinical score for the stratification of mortality risk in patients with pleural infection), complete blood count, CRP, pleural fluid characteristics (i.e., appearance, nucleated cells with differential, biochemical characteristics, isolated bacteria with their susceptibility test results), and outcome parameters (requirement for surgical intervention, inhospital and three-month mortality) were recorded for the participants of the prospective arm of the study $(15,16)$. Malignancy was recorded if it was diagnosed during the last two years from CAPI occurrence while the size of the effusion was semi-quantified according to the height of the fluid on the CXR after dividing the distance from the diaphragm to the apex of the hemithorax into four equal parts (i.e., $<25 \%, 26-50 \%, 51-75 \%,>75 \%$ ).

All pleural fluid samples, regardless their macroscopic aspect (purulent or not) or $\mathrm{pH}$, were cultured using routine methodology in blood culture bottles (for aerobic and anaerobic bacteria). The susceptibility testing was performed with the VITEK 2 (Bio Merieux) kit. Multidrug resistance (MDR) was defined as resistance to at least one agent in three or more antimicrobial categories (17). The effectiveness of every drug/drug combination was determined by the susceptibility test performed in each patient's pleural fluid culture. Treatment failure was defined as in vitro failure according to the susceptibility tests of all isolates.

\section{Statistical analysis}

Categorical variables were expressed as absolute numbers with relative frequencies while the continuous ones were summarized through their median and interquartile range (IQR) values. Multivariable logistic regression was used to identify independent predictors of infection with certain bacteria, antibiotic resistance and mortality. The models were selected using the method proposed by Collett with an alpha level of 0.05 (18). Due to the exploratory nature of the study, the final multivariable models included the variables that were considered important for the examined endpoint, irrespective of their statistical significance in the univariate analysis (11,13-17). Univariable models were also used as part of the exploratory analysis. P-values less than 0.05 were considered statistically significant. Model results were presented as ORs with $95 \%$ confidence intervals. Data were analyzed using Stata v.14.2 (Stata Corp., TX, USA).

\section{Ethics}

This study conformed to the provisions of the Declaration of Helsinki (as revised in 2013). The study was approved by the Ethics committees of "Sotiria" (Protocol number (P/N): 1527), "Evangelismos" (P/N: 31), "Sismanoglion" (P/N: 8716) and 401 General Army (P/N: 265) Hospitals in Athens, "G. Papanikolaou" Hospital in Thessaloniki (P/N: $60)$ and University Hospital of Larissa (P/N: 1643).

\section{Informed consent statement}

Although mandatory in the prospective arm, written informed consent was waived in the retrospective arm of the study. Patient information was anonymized with a code number.

\section{Results}

\section{Patients and bacterial isolates}

The study included 158 patients, 113 (72\%) of which were prospectively recruited while another 45 (28\%) were retrospectively detected (Figure S1). Patient characteristics and outcomes from the prospective cohort are presented in Table 1 while no such data was available for the retrospective cohort. The prevalence of various pleural isolates in both cohorts is shown in Table 2. The prevalence of specific isolates did not significantly differ between the two cohorts. Gram-positive cocci, especially the non-pneumococcal streptococci (approximately $40 \%$ of the cases), were the most common bacteria, followed by Gram-negative bacteria 
Table 1 Baseline demographic, clinical and radiological characteristics of patients with community-acquired parapneumonic pleural infection

\begin{tabular}{|c|c|}
\hline Characteristics & Prospective cohort $(n=113)$ \\
\hline \multicolumn{2}{|l|}{ Epidemiological data } \\
\hline Male gender (n, \%) & $80(70.8)$ \\
\hline Age (median, IQR) & $62.0(48.0-74.5)$ \\
\hline Current or ex-smokers (n, \%) & $71(62.8)$ \\
\hline Chronic heart disease (n, \%) & $23(20.4)$ \\
\hline Chronic renal failure (n, \%) & $4(3.5)$ \\
\hline Cerebrovascular disease (n, \%) & $19(16.8)$ \\
\hline Malignancy (n, \%) & $26(23.0)$ \\
\hline Diabetes mellitus (n, \%) & $14(12.4)$ \\
\hline Alcohol abuse (n, \%) & $13(11.5)$ \\
\hline Intra-venus drug use (n, \%) & $12(10.6)$ \\
\hline \multicolumn{2}{|l|}{ Clinical features at diagnosis } \\
\hline Fever (n, \%) & $78(69.0)$ \\
\hline Cough (n, \%) & $64(56.6)$ \\
\hline Chest Pain (n, \%) & $70(61.9)$ \\
\hline $\begin{array}{l}\text { Duration of symptoms in days } \\
\text { (median, IQR) }\end{array}$ & $7.0(3.0-10.0)$ \\
\hline Prior antimicrobial use (n, \%) & $32(28.3)$ \\
\hline \multicolumn{2}{|l|}{ Radiological signs (Chest X-ray) } \\
\hline Left side of effusion (n, \%) & $55(48.7)$ \\
\hline Parenchymal consolidation (n, \%) & $74(65.5)$ \\
\hline Loculated effusion (n, \%) & $57(50.4)$ \\
\hline \multicolumn{2}{|l|}{ Size of effusion (n, \%) } \\
\hline Pleurodiaphragmatic angle & $2(1.8)$ \\
\hline$<25 \%$ of the hemithorax & $37(32.7)$ \\
\hline $26-50 \%$ of the hemithorax & $42(37.2)$ \\
\hline $51-75 \%$ of the hemithorax & $25(22.1)$ \\
\hline$>75 \%$ of the hemithorax & $7(6.2)$ \\
\hline Hb Saturation (\%) (median, IQR) & $91.0(88.0-93.5)$ \\
\hline \multicolumn{2}{|l|}{ Rapid score risk } \\
\hline Low [0-2] (n, \%) & $43(38.1)$ \\
\hline Medium [3-4] (n, \%) & $47(41.6)$ \\
\hline High [5-7] (n, \%) & $22(19.5)$ \\
\hline
\end{tabular}

Table 1 (continued)
Table 1 (continued)

\begin{tabular}{|c|c|}
\hline Characteristics & Prospective cohort $(n=113)$ \\
\hline \multicolumn{2}{|l|}{ Pleural fluid characteristics } \\
\hline Purulent appearance (n, \%) & $60(53.1)$ \\
\hline $\mathrm{pH}$ (median, IQR) & $6.9(6.8-7.1)$ \\
\hline Glucose (mg/dL) (median, IQR) & $5.0(1.0-59.0)$ \\
\hline Total protein (g/dL) (median, IQR) & $3.9(3.0-4.9)$ \\
\hline $\begin{array}{l}\text { Lactate dehydrogenase (U/L), } \\
\text { (median, IQR) }\end{array}$ & $2,707(1,186-8,550)$ \\
\hline Total cell count $(/ \mu \mathrm{L})$ (median, IQR) & $15,625(3,900-43,200)$ \\
\hline $\begin{array}{l}\text { Polymorphonuclear cells (\%) } \\
\text { (median, IQR) }\end{array}$ & $80(70-92)$ \\
\hline \multicolumn{2}{|l|}{ Blood tests characteristics } \\
\hline $\begin{array}{l}\text { White cell count }(/ \mu \mathrm{L}) \text { (median, } \\
\mathrm{IQR})\end{array}$ & $18,100(12,500-24,100)$ \\
\hline $\begin{array}{l}\text { Polymorphonuclear cells (\%) } \\
\text { (median, IQR) }\end{array}$ & $86.4(80.0-91.0)$ \\
\hline Platelet count $(/ \mu \mathrm{L}),($ median, IQR) & $328,000(249,000-447,000)$ \\
\hline $\begin{array}{l}\text { C-reactive protein (mg/dL) } \\
\text { (median, IQR) }\end{array}$ & $19.8(12.6-32.4)$ \\
\hline \multicolumn{2}{|l|}{ Outcome } \\
\hline Requirement for surgery (n, \%) & $13(11.5)$ \\
\hline $\begin{array}{l}\text { Alive at } 3 \text { months after } \\
\text { hospitalization }(n, \%)\end{array}$ & $66(58.4)$ \\
\hline Died during hospitalization (n, \%) & $29(25.7)$ \\
\hline $\begin{array}{l}\text { Died within } 3 \text { months from } \\
\text { discharge }(n, \%)\end{array}$ & $7(6.2)$ \\
\hline Non-available data (n, \%) & $11(9.7)$ \\
\hline
\end{tabular}

$(10 \%)$ and anaerobes $(8 \%)$ while polymicrobial infections were identified in $14 \%$ of the cases. Approximately $26 \%$ of patients in the prospective cohort died during hospitalization while another $9.6 \%$ died within 3 months from hospital discharge. The cause of hospital mortality for all participants was the uncontrolled pleural sepsis. None of our patients had bronchopleural fistula. Two patients received fibrinolytic therapy with tissue plasminogen activator (tPA) and deoxyribonuclease (DNase). Both patients recovered. A subset of 13 patients $(11.5 \%)$ underwent surgery for the treatment of pleural sepsis. Nine of them (69\%) had medium/high RAPID score and four of 
Table 2 Bacteriology of community-acquired parapneumonic pleural infection in Greece

\begin{tabular}{|c|c|c|c|}
\hline & Retrospective cohort, (n, \%) & Prospective cohort, (n, \%) & Overall, (n, \%) \\
\hline Gram-positive bacteria & $33(73.3)$ & $79(69.9)$ & $112(70.9)$ \\
\hline Streptococcus pneumoniae & $2(4.4)$ & $22(19.5)$ & $24(15.2)$ \\
\hline Streptococcus milleri & $4(8.9)$ & $18(15.9)$ & $22(13.9)$ \\
\hline Other viridans streptococci & $4(8.9)$ & $17(15.0)$ & $21(13.3)$ \\
\hline Enterococcus spp & $6(13.3)$ & $8(7.1)$ & $14(8.9)$ \\
\hline Staphylococcus aureus & $8(17.8)$ & $7(6.2)$ & $15(9.5)$ \\
\hline Methicillin-sensitive (MSSA) & $7(15.6)$ & $7(6.2)$ & $14(8.9)$ \\
\hline Other gram positive bacteria & $0(0.0)$ & $2(1.8)$ & $2(1.3)$ \\
\hline Gram-negative bacteria & $7(15.6)$ & $9(8.0)$ & $16(10.1)$ \\
\hline Acinetobacter spp & $0(0.0)$ & $5(4.4)$ & $5(3.2)$ \\
\hline Pseudomonas aeruginosa & $1(2.2)$ & $3(2.7)$ & $4(2.5)$ \\
\hline Klebsiella spp & $5(11.1)$ & $4(3.5)$ & $9(5.7)$ \\
\hline Proteus mirabilis & $1(2.2)$ & $1(0.9)$ & $2(1.3)$ \\
\hline Other gram negative bacteria & $2(4.4)$ & $1(0.9)$ & $3(1.9)$ \\
\hline Anaerobic bacteria & $2(4.4)$ & $11(9.7)$ & $13(8.2)$ \\
\hline
\end{tabular}

them (31\%) were alcohol abusers. Streptococcus milleri group was isolated in $31 \%$ of the cases, Streptococcus pneumoniae in $23 \%$ and staphylococcus aureus in $15 \%$. No survival data were available for eleven patients who were lost from follow-up.

\section{Susceptibility testing}

To investigate the susceptibility of bacterial isolates to various antibiotics, data from both the prospective and the retrospective cohorts were examined. The prevalence of sensitivity to single antibiotics or antibiotic combinations among isolates, is presented in Table 3. The lowest (56\%) and the highest (94\%) prevalence of sensitivity were seen with penicillin $G$ and meropenem, respectively. Regarding the combinations, the highest (94\%) prevalence of sensitivity was seen with meropenem plus linezolid while similar results were found with the combinations of aminopenicillin/lactamase inhibitor plus respiratory fluoroquinolone (93\%) and ceftriaxone plus respiratory fluoroquinolone (89\%).

\section{Predictors of causative bacteria}

Several patient characteristics were connected to the isolation of specific bacteria from the pleural fluid in the univariate analyses (Table 4). Intravenous drug use (OR: 
Table 3 Prevalence of sensitivity of bacterial isolates to various antibiotics (prospective and retrospective group)

\begin{tabular}{lc}
\hline Antibiotics & $\%(95 \% \mathrm{Cl})$ \\
\hline Single antibiotics & $56.0(47.4,64.4)$ \\
Penicillin G & $66.0(57.5,73.7)$ \\
Clindamycin & $73.0(64.9,80.2)$ \\
Ceftriaxone & $82.3(74.9,88.2)$ \\
Aminopenicillin-Inhibitor & $86.5(79.8,91.7)$ \\
Fluoroquinolone & $90.1(83.9,94.5)$ \\
Piperacillin-tazobactam & $93.6(88.2,97.0)$ \\
Meropenem & $85.8(78.9,91.1)$ \\
Linezolid & \\
Antibiotic combinations & $87.9(81.4,92.8)$ \\
Aminopenicillin-inhibitor + clindamycin & $92.9(87.3,96.5)$ \\
Aminopenicillin-inhibitor + fluoroquinolone & $89.4(83.1,93.9)$ \\
Ceftriaxone + fluoroquinolone & $91.5(85.6,95.5)$ \\
Piperacillin-tazobactam + clindamycin & $92.9(87.3,96.5)$ \\
Piperacillin-tazobactam + fluoroquinolone & $94.3(89.1,97.5)$ \\
Meropenem + linezolid &
\end{tabular}

13.38) and parenchymal consolidation (OR: 6.48) were associated with infection from Streptococcus pneumoniae while cerebrovascular disease (OR: 3.15) and high blood leucocyte counts (OR: 1.89) were associated with Streptococcus milleri infection. Diabetes was associated with the presence of Enterococcus (OR: 5.07), Klebsiella (OR: 26.45) or other (e.g., Sphingomonas paucimobilis, etc.) Gram-negative bacteria (OR: 9.75). Longer duration of symptoms was linked to higher risk of Enterococcus (OR: 1.11) infection while patients with purulent pleural fluid (OR: 3.39) or malignancy (OR: 5.72) presented higher risk for infection from Gramnegative bacteria.

\section{Predictors of antibiotic resistance}

Chronic renal failure was the most common independent predictor of antibiotic resistance, associated with increased risk of resistance to four of the six antibiotic combinations tested. Diabetes, female sex and prior antimicrobial use were also independent risk factors for resistance to three, two and one combination, respectively (Table 5). In the univariate analysis, chronic renal failure, diabetes and female sex were associated with risk of resistance to multiple antibiotic combinations while prior antimicrobial use and malignancy were associated with resistance to only one combination.

\section{Predictors of mortality}

In the multivariate analyses, RAPID score (high/medium) was the most robust independent predictor of in-hospital and 3-month mortality while diabetes and high CRP were associated with 5.25- and 2.4-times higher risk for inhospital mortality, respectively (Table 6). In the univariate analyses, higher mortality was additionally observed in patients with cerebrovascular disease and lower mortality in those with normal oxygenation, absence of fever or loculated pleural effusion at presentation.

\section{Discussion}

We here investigated the prevalence of bacteria among pleural fluid isolates from patients with culture-positive, CAPI (including both purulent and non-purulent effusions), we identified their susceptibility test results along with the outcomes of the patients and examined the association of these parameters with patients' clinical characteristics. Gram-positive cocci, especially the nonpneumococcal streptococci, were the most common. Most isolates were sensitive to the combination of a respiratory fluoroquinolone with ceftriaxone or an aminopenicillin/ lactamase inhibitor. Mortality was strongly associated with medium/high RAPID score, diabetes or high CRP levels while several patient characteristics were related to the presence of certain bacteria (i.e., intravenous drug use, lung consolidation, cerebrovascular disease, high leucocyte count, diabetes, female gender, malignancy and purulent pleural fluid) or the presence of resistance to certain antibiotics (i.e., diabetes, chronic renal failure, lung consolidation, prior antibiotic use and female gender).

This study includes patients with culture-positive effusions because our primary goal was to identify the microbiology of the pleural sepsis in Greece. The diagnostic performance of any microbiology test was not studied. The bacteriologic profile of CAPI in our study, namely the dominance of streptococci (55\%) with the staphylococci, Gram-negatives and anaerobes accounting for $17.1 \%, 10.1 \%$ and $8.2 \%$ of the cases respectively, is in accordance with that of MIST1 trial where the prevalence of the same bacteria 
Table 4 Association (univariate logistic regression analysis) of clinical characteristics with certain pathogens in patients with CAPI (prospective group only)

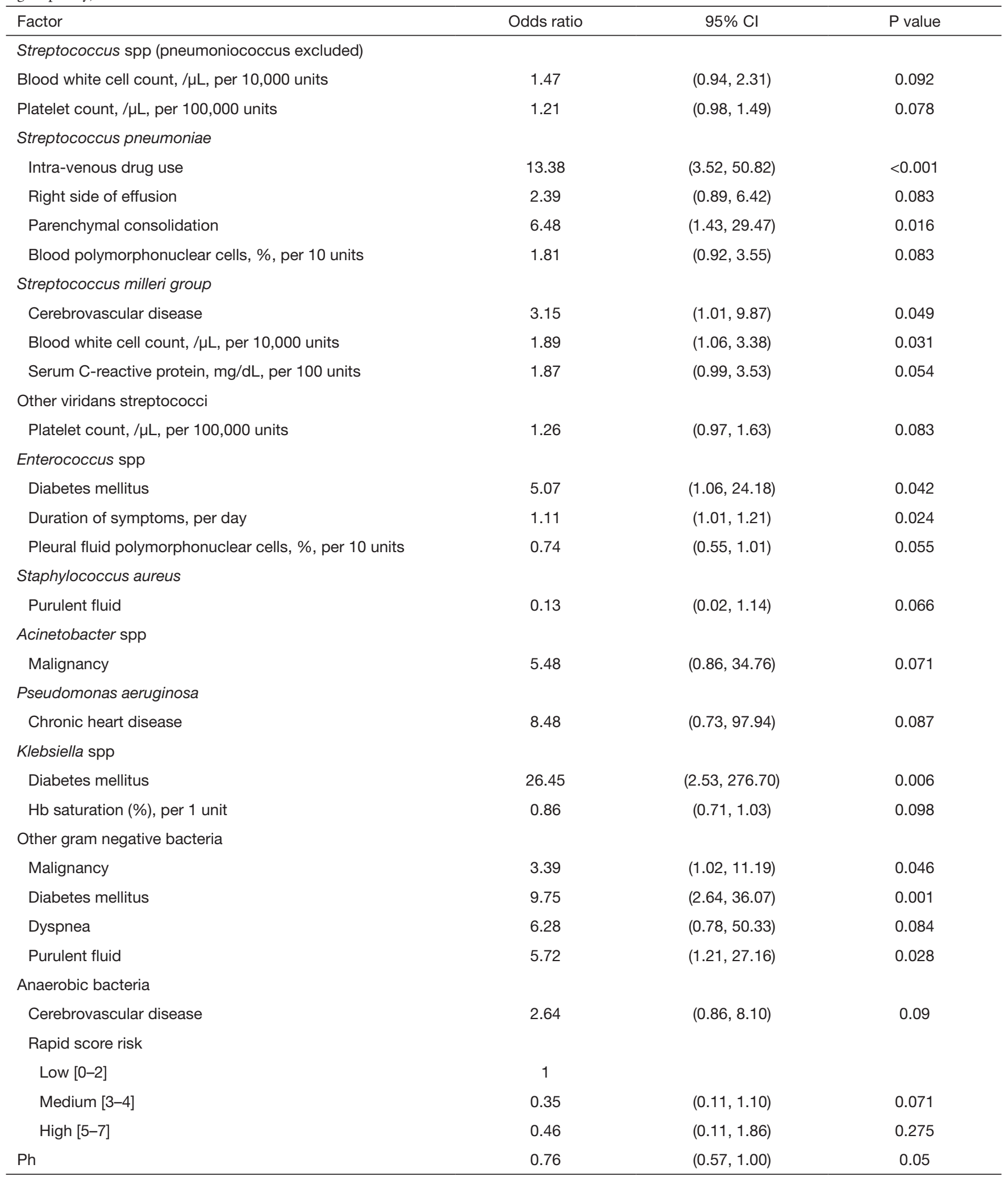

Univariate logistic regression analysis. Results are shown if $\mathrm{P}$ value $<0.10$. CAPI, community-acquired pleural infection. 
Table 5 Factors associated with resistance to antibiotic combinations commonly used in the treatment of patients with community-acquired pleural infection (prospective group only)

\begin{tabular}{|c|c|c|c|c|}
\hline & \multicolumn{2}{|c|}{ Univariate analysis } & \multicolumn{2}{|c|}{ Multivariate analysis } \\
\hline \multicolumn{5}{|c|}{ Aminopenicillin-inhibitor + clindamycin } \\
\hline Diabetes mellitus & $4.28(1.07,17.08)$ & 0.04 & $6.93(1.41,34.04)$ & 0.017 \\
\hline Parenchymal consolidation & $0.28(0.08,0.97)$ & 0.045 & $0.25(0.06,0.99)$ & 0.048 \\
\hline Chronic renal failure & $12.86(0.72,228.85)$ & $0.082^{*}$ & $92.92(2.61,306.64)$ & 0.013 \\
\hline Malignancy & $3.09(0.71,13.40)$ & $0.132^{*}$ & $4.88(0.95,24.99)$ & 0.057 \\
\hline Parenchymal consolidation & $3.36(0.64,17.54)$ & $0.151^{*}$ & $7.38(0.82,66.43)$ & 0.075 \\
\hline \multicolumn{5}{|l|}{ Ceftriaxone + fluoroquinolone } \\
\hline Diabetes mellitus & $5.85(1.39,24.72)$ & 0.016 & $7.86(1.62,38.23)$ & 0.011 \\
\hline \multicolumn{5}{|c|}{ Piperacillin-tazobactam + clindamycin } \\
\hline Chronic renal failure & $11.12(0.63,195.17)$ & $0.099^{*}$ & $21.67(1.07,436.82)$ & 0.045 \\
\hline Prior antimicrobial use & $3.75(0.92,15.21)$ & $0.064^{*}$ & $4.92(1.09,22.31)$ & 0.039 \\
\hline \multicolumn{5}{|c|}{ Piperacillin-tazobactam + fluoroquinolone } \\
\hline Chronic renal failure & $12.86(0.72,228.25)$ & $0.082^{*}$ & $92.92(2.61,3,306.6)$ & 0.013 \\
\hline Malignancy & $3.09(0.71,13.40)$ & $0.132^{*}$ & $4.88(0.95,24.99)$ & 0.057 \\
\hline Parenchymal consolidation & $3.36(0.64,17.54)$ & $0.151^{*}$ & $7.38(0.82,66.43)$ & 0.075 \\
\hline
\end{tabular}

*, results considered important for resistance to antibiotic combinations were used in multivariate analysis irrespective of the level of statistical significance and are presented in the univariate analysis.

was $52 \%, 10.4 \%, 8.6 \%$ and $19.9 \%$, respectively (2). In another study, Hassan et al. had found that Viridans streptococci were the most common microbes in temperate regions. Also, the same study highlighted the pivotal role that each geographical location has on the expected causative pathogen (19). However, the higher $(19.9 \%$ versus $8.2 \%)$ prevalence of anaerobes in MIST1 is probably due to the increased sensitivity of the bacterial detection approach, which used nucleic acid amplification tests complementary to cultures for this purpose (2). In all studies, Hassan et al., MIST and the current study, the prevalence of nonpneumococcal streptococci surpassed that of Streptococcus pneumoniae. In contrast, Falguera et al. found Streptococcus pneumoniae to be the most common pathogen among 261 CAPI patients in Spain (13). This, along with the reported increased prevalence of Gram-negative bacteria, especially Klebsiella spp, among CAPI patients in Taiwan, highlights the importance of considering regional differences in the bacteriology of the disease when selecting antibiotics $(6,20)$. 
Table 6 Factors predicting mortality in patients with community-acquired parapneumonic pleural infection (prospective group)

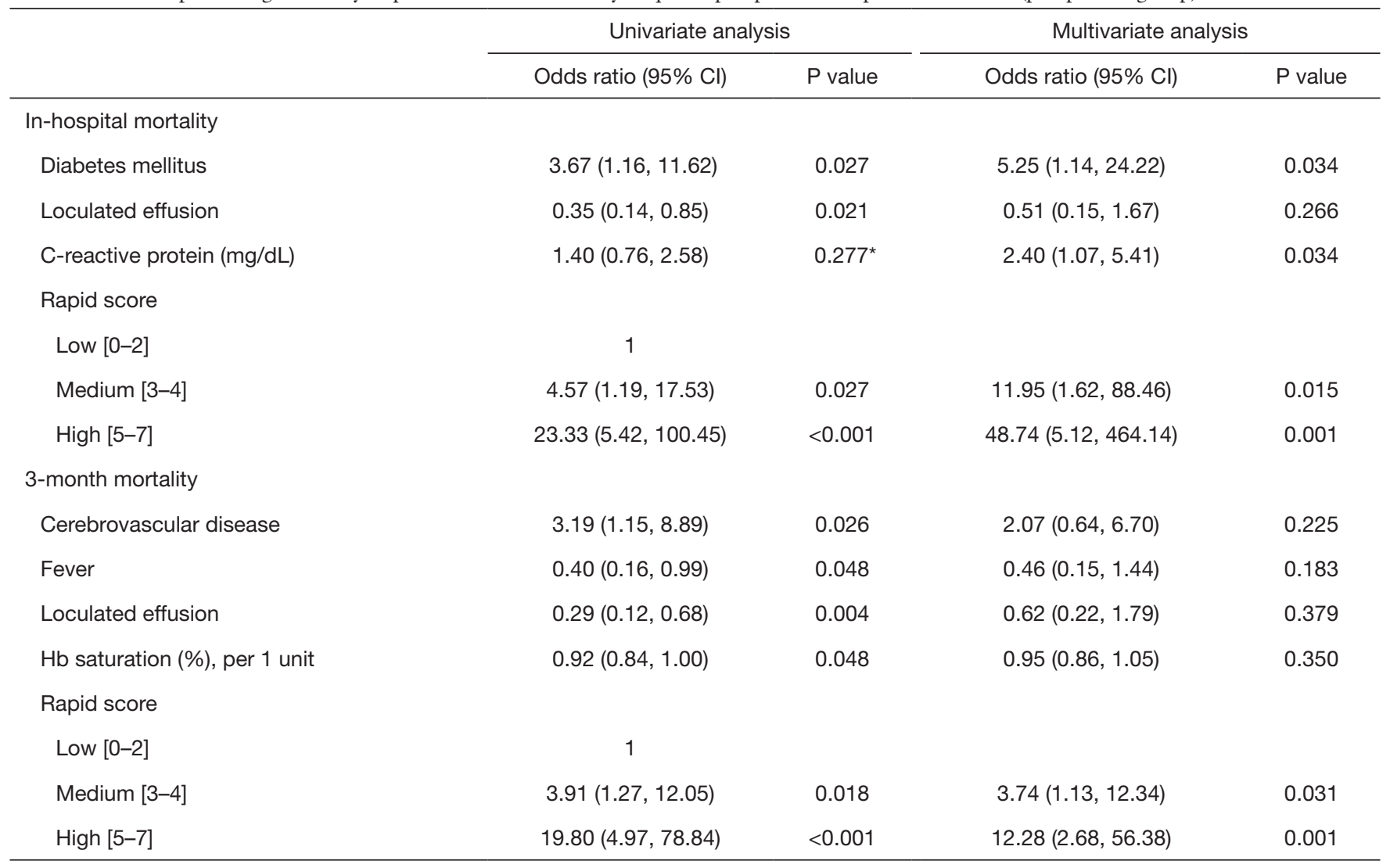

${ }^{*}$, results considered important for the clinical outcome are used in multivariate analysis irrespective of the level of statistical significance and are presented in the univariate analysis.

We here also examined whether certain patient characteristics could predict the causative bacteria. Diabetes was an independent risk factor for infection from Enterococcus and Gram-negative bacteria, especially Klebsiella. Cerebrovascular disease was related to infection from Streptococcus milleri and anaerobes while the presence of parenchymal consolidation or intravenous drug use was associated with Streptococcus pneumoniae infection. The link between diabetes and Klebsiella-associated CAPI has been also reported by Lin et al., who additionally found cirrhosis and lung cancer to be independent risk factors for this entity while the association of cerebrovascular disease with infection by bacteria of the oropharyngeal flora, such as Streptococcus milleri and anaerobes, adds to the evidence that aspiration plays a key role in the development of pleural infection in such patients $(1,20)$. The fact that only Streptococcus pneumoniae was significantly associated with the presence of consolidation on chest X-ray, not only assists the selection of appropriate antibiotics in such patients but also agrees with the observation that CAPI may occur in the absence of significant parenchymal disease, most commonly by non-pneumococcal bacteria $(1,21)$.

Finally, we recorded the susceptibility test results of the isolates and investigated whether antibiotic resistance could be predicted by patient clinical characteristics. We found that none of the antibiotic combinations in our study was $100 \%$ effective, which implies the presence of MDR strains even in the community-acquired infections. Most of the patients with MDR-CAPI had a history of malignancy, which agrees with previous observations that cancer patients are frequently colonized with and are susceptible to infections by MDR Gram-negative bacteria $(22,23)$. This, along with the elevated proportion ( $23 \%$ of all patients) of cancer patients in our study may explain the increased 3 -month mortality in our population compared to that in MIST1 (32\% versus 16\%) (8). Nevertheless, the isolates from $93 \%$ and $89 \%$ of the patients were sensitive to the combination of an aminopenicillin/lactamase inhibitor or 
ceftriaxone with a respiratory fluoroquinolone, respectively. Independent risk factors for the presence of resistance to these antibiotic regimens included chronic renal failure and diabetes, which should be considered when selecting antibiotics in such patients.

The research for mortality predictors in CAPI is limited. Recently, Rahman et al. evaluated patients with both hospital- and CAPI to develop a clinical score (i.e., RAPID score) for this purpose (16) which was recently validated prospectively (15). In our study, RAPID score was prospectively validated in a pure CAPI, Greek population and confirmed its reliability to predict mortality in this setting while diabetes and CRP were also found useful for the same purpose. Diabetes has been previously reported to be a common co-morbidity that aggravates the prognosis of these patients by predisposing to infection by Gramnegative bacteria and fungi while the connection between high CRP and increased mortality risk is in accordance with previous studies reporting that CRP $>79-100 \mathrm{mg} / \mathrm{L}$ can accurately discriminate complicated from uncomplicated parapneumonic effusions (24-27). Overall, we validated RAPID score as a robust outcome predictor in CAPI, Greek patients. Also, univariate analysis revealed two more clinical parameters (i.e., diabetes and CRP) that require further investigation in large multicenter studies to confirm their usefulness in predicting the outcome of the disease.

This study has limitations. First, the fact that data for retrospective and prospective groups were analyzed together to describe the microbiology and the resistance patterns, might have insert a bias. However, we believe that this bias was not important, since the prevalence of various bacteria between the two cohorts do not differ significantly. In addition, the combined group was used only for descriptive purposes and analytical statistics to identify predictive factors for drug resistance or outcomes were performed in the prospective group only. Second, CAPI diagnosis was based solely on positive pleural fluid cultures, which means that patients with bacteria recovered from blood or sputum were not included, limiting our sample size. Yet, this study design warrants the inclusion of patients with definite pleural infection, which is the reason for being previously used in similar studies. Third, the use of more sensitive isolation methods, such as nucleic acid amplification tests or pleural biopsy cultures would have increased the number of included patients but such methods were not in routine clinical use during the study period $(28,29)$.

Finally, one could question the relevance of our results for populations outside Greece. Accepting the geographic variation in the bacteriology of the disease and considering that this is an inherent limitation of similar bacteriologic studies that cannot be easily conducted in an international setting, we believe that the importance of the present study lies indeed in the addition of regional data to the global map of CAPI bacteriology.

\section{Conclusions}

This study found streptococci, especially the nonpneumococcal ones, to be the most common isolates from the pleural fluid of CAPI-patients, and demonstrated that common antibiotic regimens, based on local antibiotic policies, are highly effective in the treatment of this infection. RAPID score was prospectively validated in Greek population and its robustness in the prediction of mortality in these patients was confirmed. Finally, additional risk factors of mortality and antibiotic resistance warranting further investigation were detected.

\section{Acknowledgments}

We are particularly grateful to the following Institutions who have given us help on recruitment of patients of our study: $-1^{s t}, 5^{t h}, 7^{\text {th }}$ and 10th Respiratory Medicine Department, Athens Chest Hospital "Sotiria", Chair Persons Dr. N. Koulouris, Dr. K. Dimakou, Dr. M. Gaga, Dr. E. Kainis, 1 st and $2^{\text {nd }}$ Department of Pulmonary Disease, "Sismanoglion" General Hospital, Chair Persons Dr. X. Tsiafaki, Dr. V. Filaditaki. Also, we would like to thank the participants of the study as without them this study would not be possible.

Funding: None.

\section{Footnote}

Reporting Checklist: The authors have completed the STROBE reporting checklist. Available at http://dx.doi. org/10.21037/jtd-20-2786

Data Sharing Statement: Available at http://dx.doi. org/10.21037/jtd-20-2786

Peer Review File: Available at http://dx.doi.org/10.21037/jtd20-2786

Conflicts of Interest: All authors have completed the ICMJE uniform disclosure form (available at http://dx.doi. org/10.21037/jtd-20-2786). The authors have no conflicts of 
interest to declare.

Ethical Statement: The authors are accountable for all aspects of the work in ensuring that questions related to the accuracy or integrity of any part of the work are appropriately investigated and resolved. This study conformed to the provisions of the Declaration of Helsinki (as revised in 2013). Also, it was approved by the Ethics committees of "Sotiria" (Protocol number (P/N): 1527), "Evangelismos" (P/N: 31), "Sismanoglion" (P/N: 8716) and 401 General Army (P/N: 265) Hospitals in Athens, "G. Papanikolaou" Hospital in Thessaloniki (P/N: 60) and University Hospital of Larissa (P/N: 1643). Although mandatory in the prospective arm, written informed consent was waived in the retrospective arm of the study. Patient information was anonymized with a code number.

Open Access Statement: This is an Open Access article distributed in accordance with the Creative Commons Attribution-NonCommercial-NoDerivs 4.0 International License (CC BY-NC-ND 4.0), which permits the noncommercial replication and distribution of the article with the strict proviso that no changes or edits are made and the original work is properly cited (including links to both the formal publication through the relevant DOI and the license). See: https://creativecommons.org/licenses/by-nc-nd/4.0/.

\section{References}

1. Corcoran JP, Wrightson JM, Belcher E, et al. Pleural infection: past, present, and future directions. Lancet Respir Med 2015;3:563-77.

2. Maskell NA, Batt S, Hedley EL, et al. The bacteriology of pleural infection by genetic and standard methods and its mortality significance. Am J Respir Crit Care Med 2006;174:817-23.

3. Finley C, Clifton J, Fitzgerald JM, et al. Empyema: an increasing concern in Canada. Can Respir J 2008;15:85-9.

4. Byington CL, Spencer LY, Johnson TA, et al. An epidemiological investigation of a sustained high rate of pediatric parapneumonic empyema: risk factors and microbiological associations. Clin Infect Dis 2002;34:434-40.

5. Byington CL, Samore MH, Stoddard GJ, et al. Temporal trends of invasive disease due to Streptococcus pneumoniae among children in the intermountain west: emergence of nonvaccine serogroups. Clin Infect Dis 2005;41:21-9.

6. Davies HE, Davies RJO, Davies CWH, et al. Management of pleural infection in adults: British Thoracic Society Pleural Disease Guideline 2010. Thorax 2010;65 Suppl 2:ii41-53.

7. Colice GL, Curtis A, Deslauriers J, et al. Medical and surgical treatment of parapneumonic effusions : an evidence-based guideline. Chest 2000;118:1158-71.

8. Rahman NM, Maskell NA, West A, et al. Intrapleural use of tissue plasminogen activator and DNase in pleural infection. N Engl J Med 2011;365:518-26.

9. Suchar AM, Zureikat AH, Glynn L, et al. Ready for the frontline: is early thoracoscopic decortication the new standard of care for advanced pneumonia with empyema? Am Surg 2006;72:688-92; discussion 692.

10. Chung JH, Lee SH, Kim KT, et al. Optimal timing of thoracoscopic drainage and decortication for empyema. Ann Thorac Surg 2014;97:224-9.

11. Kim SK, Kang CU, Song SH, et al. Factors predictive of the failure of medical treatment in patients with pleural infection. Korean J Intern Med 2014;29:603-12.

12. Maskell NA, Davies CWH, Nunn AJ, et al. U.K. Controlled trial of intrapleural streptokinase for pleural infection. N Engl J Med 2005;352:865-74.

13. Falguera $M$, Carratalà $J$, Bielsa $S$, et al. Predictive factors, microbiology and outcome of patients with parapneumonic effusion. Eur Respir J 2011;38:1173-9.

14. Meyer CN, Armbruster K, Kemp M, et al. Pleural infection: a retrospective study of clinical outcome and the correlation to known etiology, co-morbidity and treatment factors. BMC Pulm Med 2018;18:160.

15. Corcoran JP, Psallidas I, Gerry S, et al. Prospective validation of the RAPID clinical risk prediction score in adult patients with pleural infection: the PILOT study. Eur Respir J 2020;56:2000130.

16. Rahman NM, Kahan BC, Miller RF, et al. A clinical score (RAPID) to identify those at risk for poor outcome at presentation in patients with pleural infection. Chest 2014;145:848-55.

17. Magiorakos AP, Srinivasan A, Carey RB, et al. Multidrugresistant, extensively drug-resistant and pandrug-resistant bacteria: an international expert proposal for interim standard definitions for acquired resistance. Clin Microbiol Infect 2012;18:268-81.

18. Collett D. Modelling survival data in medical research. Boston, MA: Springer, 1994.

19. Hassan M, Cargill T, Harriss E, et al. The microbiology of pleural infection in adults: a systematic review. Eur Respir J 2019;54:1900542.

20. Lin YT, Chen TL, Siu LK, et al. Clinical and 
microbiological characteristics of community-acquired thoracic empyema or complicated parapneumonic effusion caused by Klebsiella pneumoniae in Taiwan. Eur J Clin Microbiol Infect Dis 2010;29:1003-10.

21. Light RW. Parapneumonic effusions and empyema. Proc Am Thorac Soc 2006;3:75-80.

22. Monteiro R, Alfaro TM, Correia L, et al. Lung abscess and thoracic empyema: retrospective analysis in an internal medicine department. Acta Med Port 2011;24 Suppl 2:229-40.

23. Hess AS, Kleinberg M, Sorkin JD, et al. Prior colonization is associated with increased risk of antibiotic-resistant Gram-negative bacteremia in cancer patients. Diagn Microbiol Infect Dis 2014;79:73-76.

24. Smith JA, Mullerworth MH, Westlake GW, et al. Empyema thoracis: 14-year experience in a teaching center. Ann Thorac Surg 1991;51:39-42.

Cite this article as: Iliopoulou M, Skouras V, Psaroudaki Z, Makarona M, Vogiatzakis E, Tsorlini E, Katsifa E, Spyratos D, Siopi D, Kotsiou O, Xitsas S, Martsoukou M, Sigala I, Kalomenidis I. Bacteriology, antibiotic resistance and risk stratification of patients with culture-positive, communityacquired pleural infection. J Thorac Dis 2021;13(2):521-532. doi: 10.21037/jtd-20-2786
25. Porcel JM. Distinguishing complicated from uncomplicated parapneumonic effusions. Curr Opin Pulm Med 2015;21:346-51.

26. Porcel JM, Bielsa S, Esquerda A, et al. Pleural fluid C-reactive protein contributes to the diagnosis and assessment of severity of parapneumonic effusions. Eur J Intern Med 2012;23:447-50.

27. Skouras V, Boultadakis E, Nikoulis D, et al. Prognostic value of $\mathrm{C}$-reactive protein in parapneumonic effusions. Respirology 2012;17:308-14.

28. Nielsen J, Meyer CN, Rosenlund S. Outcome and clinical characteristics in pleural empyema: a retrospective study. Scand J Infect Dis 2011;43:430-5.

29. Psallidas I, Kanellakis NI, Bhatnagar R, et al. A Pilot Feasibility Study in Establishing the Role of UltrasoundGuided Pleural Biopsies in Pleural Infection (The AUDIO Study). Chest 2018;154:766-72. 


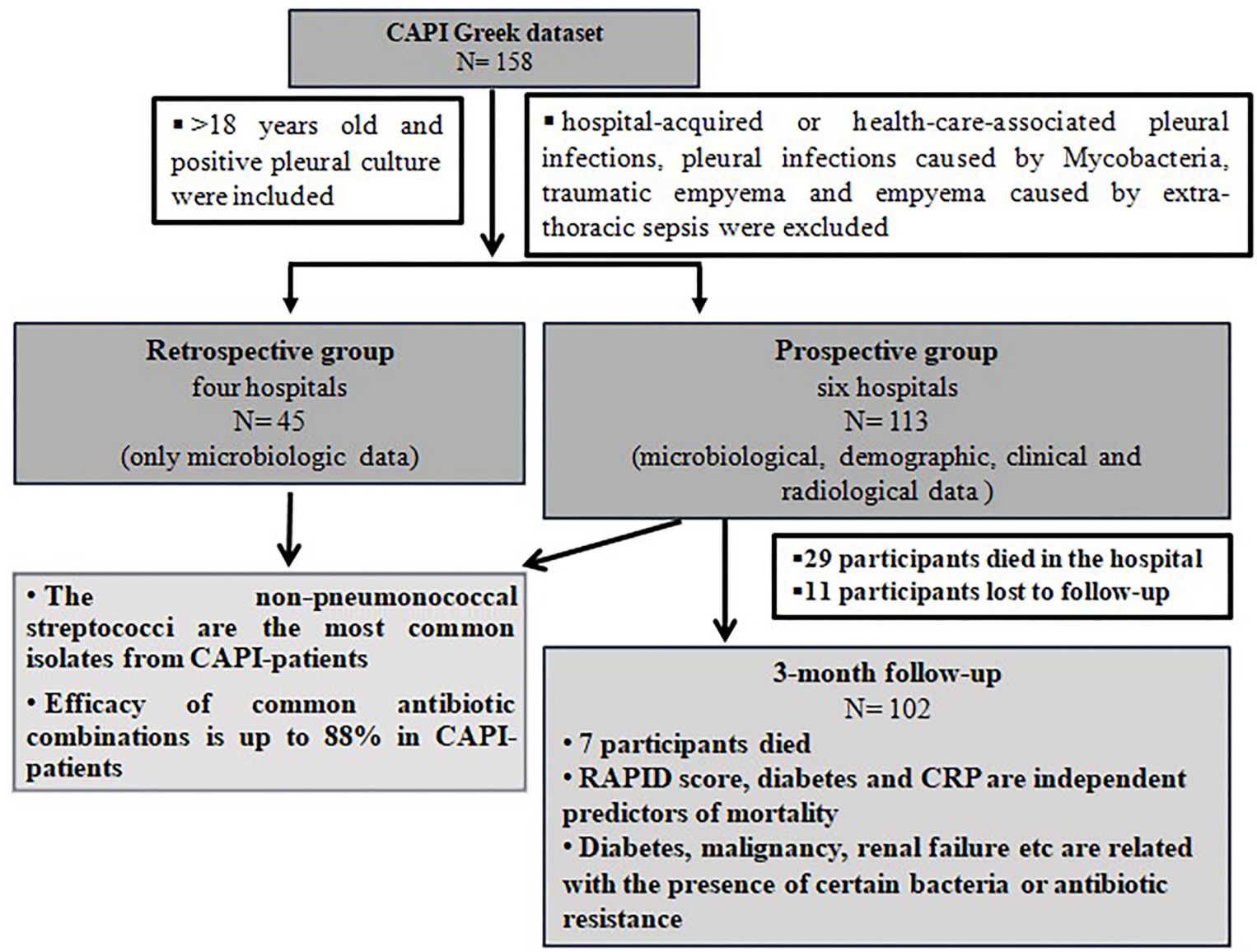

Figure S1 Flowchart describing the study's pipeline, data availability for each cohort and the main results. 